Richard C. Brown, Department of Mathematics, University of Alabama, Tuscaloosa, AL 35487-0350, USA, e-mail: dicbrown@@ua1vm.ua.edu Don B. Hinton, Department of Mathematics, University of Tennessee, Knoxville, TN 37996-1300, e-mail: hinton@@novell.math.utk.edu Alois Kufner, Mathematical Institute, Czech Academy of Sciences, Žitná 25, 11567 Prague 1, Czech Republic, e-mail: kufner@@earn.cvut.cz

\title{
SUFFICIENT CONDITIONS FOR THREE WEIGHT SUM INEQUALITIES IN LEBESGUE SPACES
}

\begin{abstract}
Conditions (in terms of integrals of the weights) are derived, under which the weighted $L^{q}$-norm of the $j$-th order derivative of the function $u$ can be estimated by the sum of the weighted $L^{r}$-norm of $u$ and of the weighted $L^{p}$-norm of its $m$-th order derivative, $j<m$. All mutual positions of the parameters, $p, q, r$ are admissible.
\end{abstract}

\section{Introduction}

The aim of this paper is to describe conditions under which a three weightinequality of the form

$$
\left\|u^{(j)}\right\|_{q, W} \leq C\left(\|u\|_{r, W_{0}}+\left\|u^{(m)}\right\|_{p, W_{m}}\right)
$$

holds for a certain class of (sufficiently smooth) functions $u$ with a constant $C>0$ independent of $u$, i.e., to characterize the weights $W, W_{m}, W_{0}$ (= measurable positive a.e. functions) for which (1.1) is true for various choices of the parameters $p, q, r \in(1, \infty)$.

Key Words: weighted inequalities, weighted Sobolev space, interpolation inequalities

Mathematical Reviews subject classification: Primary: 26D10, 46E30

Received by the editors May 8, 1996

Supported in part by a grant from the Science Alliance Fund of the University of Tennessee and in part by the Grant Agency of the Czech Republic, grant Nr 201/94/1066 and by the Grant Agency of the Academy of Sciences of Czech Republic, Grant Nr 1019506

${ }^{*}$ The third author also wishes to thank the Department of Mathematics of the University of Tennessee for its support and hospitality. 
We will consider the one-dimensional case, i.e., $u=u(t)$ is a function defined on an interval $I=(a, b)$ with $-\infty \leq a<b \leq \infty$ and we use the notation

$$
\|u\|_{s, V}=\left(\int_{a}^{b}|u(t)|^{s} V(t) d t\right)^{\frac{1}{s}}, \quad s>1
$$

to signify the norm of $u$ in the weighted Lebesgue space $L^{s}(I ; V)$. Further we suppose that the integers $j, m$ satisfy $0 \leq j<m$.

There are many results concerning inequalities of the type (1.1) even in the higher dimensional case. Let us mention, e.g. the papers of P. I. Lizorkin and M. Otelbaev [9], [10], Brown and Hinton [1], [2], [3], [4], [5] as well as the book of V. Maz'ja [11] where for the special case $j=0, m=1$ necessary and sufficient conditions are given under which an analogue of (1.1) holds for functions which are defined in a domain $\Omega \subset \mathbb{R}^{N}, N>1$, or the recent papers of R. Oinarov [12] and B. Curgus and T. Read [6] dealing with the one-dimensional case, again for the special case of (1.1) for which $p=r$. In the last two papers, necessary and sufficient conditions are given, for $p \leq q$ in [6] and for $p \leq q$ as well as for $p>q$ in [13]. Unfortunately the conditions described in all the papers we have mentioned are complicated and not easy to verify. Moreover the problem of finding conditions for (1.1) when $j>0$ which are both necessary and sufficient is unsolved for general weights $W, W_{m}, W_{0}$.

The approach described in this paper gives for the most part only sufficient conditions, but for every choice of the parameters $p, q, r$ and also for higher values of $j<m$. Moreover the conditions given here can be easily verified and examples are given showing that the conditions are "not far" from the necessary ones.

The method used here is rather elementary. We start with a certain type of unweighted interpolation inequality and then use both Hölder's inequality and various Hardy inequalities; the conditions that allow us to apply Hardy's inequality are then in fact the conditions guaranteeing the validity of (1.1). In the following two sections we will describe two approaches depending on two choices of the basic interpolation inequality.

\section{The First Approach}

$J \subset \mathbb{R}$ be a compact interval of length $|J|$ and let

$$
A C^{k}(J)=\left\{u: u^{(k)} \text { is absolutely continuous on } J\right\} .
$$


Then if $u=u(t) \in A C^{m-1}(J)$ the following inequality holds (see, e.g., [1, Lemma 2.1]):

$$
\left|u^{(j)}(t)\right| \leq C|J|^{-(j+1)} \int_{J}|u(s)| d s+|J|^{m-(j+1)} \int_{J}\left|u^{(m)}(s)\right| d s
$$

with a positive constant $C$ depending only on the integers $j$ and $m, 0 \leq j<m$ and for every $t \in J$.

First we will consider functions $u=u(t)$ defined on an interval $I=[0, b)$ which is bounded from below: $0<b \leq \infty$. Here we require that $u \in A C_{\mathrm{loc}}^{m-1}(I)$ where

$$
A C_{\mathrm{loc}}^{k}(I)=\left\{u: u \in A C^{k}(J) \text { for every compact subinterval } J \in I\right\} .
$$

Using (2.1) for the finite interval $J=[0, t]$ we obtain the inequality

$$
\left|u^{(j)}(t)\right| \leq C t^{-(j+1)} \int_{0}^{t}|u(s)| d s+t^{m-(j+1)} \int_{0}^{t}\left|u^{(m)}(s)\right| d s .
$$

Now let $r_{0}>1, r_{m}>1$ be two auxiliary parameters and $w_{0}, w_{m}$ two auxiliary weight functions. Then Hölder's inequality yields

$$
\begin{aligned}
\int_{0}^{t}|u(s)| d s & =\int_{0}^{t}|u(s)| w_{0}^{\frac{1}{r_{0}}}(s) w_{0}^{-\frac{1}{r_{0}}}(s) d s \\
& \leq\left(\int_{0}^{t}|u(s)|^{r_{0}} w_{0}(s) d s\right)^{\frac{1}{r_{0}}}\left(\int_{0}^{t} w_{0}^{1-r_{0}^{\prime}}(s) d s\right)^{\frac{1}{r_{0}^{\prime}}}
\end{aligned}
$$

and

$$
\begin{aligned}
\int_{0}^{t}\left|u^{(m)}(s)\right| d s \leq & \left(\int_{0}^{t}\left|u^{(m)}(s)\right|^{r_{m}} w_{m}(s) d s\right)^{\frac{1}{r_{m}}} \\
& \times\left(\int_{0}^{t} w_{m}^{1-r_{m}^{\prime}}(s) d s\right)^{\frac{1}{r_{m}^{\prime}}}
\end{aligned}
$$

where as usual $r_{i}^{\prime}=r_{i}\left(r_{i}-1\right)^{-1}$ for $i=0, m$, so that $-r_{i}^{\prime} / r_{i}=1 /\left(1-r_{i}\right)=1-r_{i}^{\prime}$.

Next we proceed in the following way: First we use the estimates (2.3) and (2.4) in the right hand side of (2.2), and in the resulting inequality we take the $q$-th power of both sides with $q>1$ and use in the right hand side the elementary inequality $(A+B)^{q} \leq 2^{q-1}\left(A^{q}+B^{q}\right)$. Secondly we multiply both sides of the resulting inequality by the weight function $W(t)$ and integrate the resulting inequality with respect to $t$ from 0 to $b$. This finally yields that

$$
\int_{0}^{b}\left|u^{(j)}(t)\right|^{q} W(t) d t \leq C_{1}\left(\mathcal{J}_{1}+\mathcal{J}_{2}\right)
$$


where $C_{1}=2^{q-1} \max \{C, 1\}$ and

$$
\begin{aligned}
\mathcal{J}_{1}=\int_{0}^{b} t^{-(j+1) q} W(t) & \left(\int_{0}^{t} w_{0}^{1-r_{0}^{\prime}}(s) d s\right)^{\frac{q}{r_{0}^{\prime}}} \\
& \times\left(\int_{0}^{t}|u(s)|^{r_{0}} w_{0}(s) d s\right)^{\frac{q}{r_{0}}} d t \\
\mathcal{J}_{2}=\int_{0}^{b} t^{(m-j-1) q} W(t) & \left(\int_{0}^{t} w_{m}^{1-r_{m}^{\prime}}(s) d s\right)^{\frac{q}{r_{m}^{\prime}}} \\
\times & \left(\int_{0}^{t}\left|u^{(m)}(s)\right|^{r_{m}} w_{m}(s) d s\right)^{\frac{q}{r_{m}}} d t .
\end{aligned}
$$

Now we estimate $\mathcal{J}_{1}$ and $\mathcal{J}_{2}$ using the Hardy inequality

$$
\int_{0}^{b} H(t)\left(\int_{0}^{t} U(s) d s\right)^{\tilde{q}} d t \leq C_{0}\left(\int_{0}^{b} K(t) U^{\tilde{p}}(t) d t\right)^{\frac{\tilde{q}}{\tilde{p}}}
$$

which holds for non-negative functions $U$ if and only if the weight functions $H$ and $K$ satisfy - for $1 \leq \tilde{p} \leq \tilde{q}<\infty$ - the condition

$$
B(x)=\left(\int_{x}^{b} H(t) d t\right)^{\frac{1}{\bar{q}}}\left(\int_{0}^{x} K^{1-\tilde{p}^{\prime}}(t) d t\right)^{\frac{1}{\tilde{p}^{\prime}}} \leq B<\infty .
$$

(For details, see e.g. B. Opic and A. Kufner [13, Theorem 1.14].)

Suppose now $p, q, r$ satisfy

$$
q \geq \max \{p, r\}>1
$$

that the auxiliary parameters $r_{0}, r_{m}$ satisfy

$$
1<r_{0}<r, \quad 1<r_{m}<p
$$

and let $W_{0}, W_{m}$ be (given) weight functions on $I$.

(a) The structure of (2.6) suggests that we should use Hardy's inequality (2.8) for the special choice

$$
\begin{aligned}
& \tilde{q}=\frac{q}{r_{0}}, \quad \tilde{p}=\frac{r}{r_{0}} \\
& H(t)=t^{-(j+1) q} W(t)\left(\int_{0}^{t} w_{0}^{1-r_{0}^{\prime}}(s) d s\right)^{\frac{q}{r_{0}^{\prime}}} \\
& K(t)=W_{0}(t) w_{0}^{-\tilde{p}}(t) \\
& U(t)=|u(t)|^{r_{0}} w_{0}(t)
\end{aligned}
$$


i.e.,

$$
K(t) U^{\tilde{p}}(t)=W_{0}(t) w_{0}^{-\tilde{p}}(t)|u(t)|^{\tilde{p} r_{0}} w_{0}^{\tilde{p}}(t)=W_{0}(t)|u(t)|^{r} .
$$

We obtain that

$$
\mathcal{J}_{1} \leq C_{0}\left(\int_{0}^{b}|u(t)|^{r} W_{0}(t) d t\right)^{\frac{q}{r}}
$$

if and only if the function

$$
\begin{aligned}
B_{1}(x)= & \left(\int_{x}^{b} t^{-(j+1) q} W(t)\left(\int_{0}^{t} w_{0}^{1-r_{0}^{\prime}}(s) d s\right)^{\frac{q}{r_{0}^{\prime}}} d t\right)^{\frac{r_{0}}{q}} \\
& \times\left(\int_{0}^{x} W_{0}^{-\frac{r_{0}}{r-r_{0}}}(s) w_{0}^{\frac{r}{r-r_{0}}}(s) d s\right)^{\frac{r-r_{0}}{r}}
\end{aligned}
$$

is bounded on $I$. Notice that $B_{1}(x)$ is the function $B(x)$ from (2.9) for our special choice (2.12) of $H$ and $K$. Because of (2.10) and (2.11) $1<\tilde{p} \leq \tilde{q}$ and we have that

$$
\tilde{p}^{\prime}=\frac{\tilde{p}}{\tilde{p}-1}=\frac{r}{r-r_{0}}, \quad 1-\tilde{p}^{\prime}=-\frac{r_{0}}{r-r_{0}}, \quad \tilde{p}\left(1-\tilde{p}^{\prime}\right)=-\tilde{p}^{\prime} .
$$

(b) The structure of (2.7) suggests that we use Hardy's inequality (2.8) for the choice

$$
\begin{aligned}
\tilde{q} & =\frac{q}{r_{m}} \quad \tilde{p}=\frac{p}{r_{m}} \\
H(t) & =t^{(m-j-1) q} W(t)\left(\int_{0}^{t} w_{m}^{1-r_{m}^{\prime}}(s) d s\right)^{\frac{q}{r_{m}^{\prime}}} \\
K(t) & =W_{m}(t) w_{m}^{-\tilde{p}}(t) \\
U(t) & =\left|u^{(m)}(t)\right|^{r_{m}} w_{m}(t) .
\end{aligned}
$$

This yields in the same way that

$$
\mathcal{J}_{2} \leq C_{0}\left(\int_{0}^{b}\left|u^{(m)}(t)\right|^{p} W_{m}(t) d t\right)^{\frac{q}{p}}
$$

if and only if the function

$$
\begin{aligned}
B_{2}(x)= & \left(\int_{x}^{b} t^{(m-j-1) q} W(t)\left(\int_{0}^{t} w_{m}^{1-r_{m}^{\prime}}(s) d s\right)^{\frac{q}{r_{m}^{\prime}}} d t\right)^{\frac{r_{m}}{q}} \\
& \times\left(\int_{0}^{x} W_{m}^{-\frac{r_{m}}{p-r_{m}}}(s) w_{m}^{\frac{p}{p-r_{m}}}(s) d s\right)^{\frac{p-r_{m}}{p}}
\end{aligned}
$$


is bounded on $I$.

Using the notation from (1.2), it follows from (2.5), (2.13) and (2.16) that

$$
\left\|u^{(j)}\right\|_{q, W}^{q} \leq C_{1} C_{0}\left(\|u\|_{r, W_{0}}^{q}+\left\|u^{(m)}\right\|_{p, W_{m}}^{q}\right),
$$

and from this inequality, the inequality (1.1) follows immediately since for $q>1$ we have $\left(A^{q}+B^{q}\right)^{1 / q} \leq A+B$.

The following Theorem summarizes our results:

Theorem 2.1 Suppose that the numbers $p, q, r>1$ satisfy

$$
q \geq \max \{p, r\} .
$$

Let $m, j$ be integers $0 \leq j<m$ and let $W, W_{0}$ and $W_{m}$ be weight functions on $I=[0, b)$. Suppose that for parameters $r_{0}, r_{m}$ with

$$
1<r_{0}<r, \quad 1<r_{m}<p,
$$

and for auxiliary weight functions $w_{0}, w_{m}$, the functions $B_{1}(x)$ and $B_{2}(x)$ from the formulas (2.14) and (2.17), respectively are bounded on $[0, b)$. Then the inequality (1.1) holds for every function $u \in W^{m ; p, r}\left(I ; W_{0}, W_{m}\right)$ where

$$
W^{m ; p, r}\left(I ; W_{0}, W_{m}\right):=\left\{u \in A C_{l o c}^{m-1}(I):\|u\|_{r, W_{0}}, \quad\left\|u^{(m)}\right\|_{p, W_{m}}<\infty\right\}
$$

with a constant $C$ independent of $u$.

Remark 2.1 (A slight weakening the conditions (2.19)). We can also suppose that

$$
1<r_{0}=r \quad \text { and } / \text { or } \quad 1<r_{m}=p .
$$

In this case, the number $\tilde{p}$ in the Hardy inequality (2.8) chosen according to (2.12) and/or (2.15), is equal to one, and for $\tilde{p}=1$, the necessary and sufficient condition (2.9) has to be modified to the form

$$
B^{*}(t)=\left(\int_{t}^{b} H(s) d s\right)^{\frac{1}{\bar{q}}} \underset{s \in[0, t)}{\operatorname{ess} \sup K(s)^{-1} \leq B^{*}<\infty .}
$$

Consequently, if we choose $r_{0}=r$, the condition (2.14) that $B_{1}(x)$ should be bounded has to be replaced by the condition that

$$
B_{1}^{*}(x)=\left(\int_{x}^{b} t^{-(j+1) q} W(t)\left(\int_{0}^{t} w_{0}^{1-r^{\prime}}(s) d s\right)^{\frac{q}{r^{\prime}}} d t\right)^{\frac{r}{q}} \underset{s \in[0, x)}{\operatorname{ess} \sup } \frac{w_{0}(s)}{W_{0}(s)}
$$

be bounded on $I$, since, due to $(2.12), K(t)=W_{0}(t) w_{0}^{-1}(t)$. 
We proceed in a similar way for the case $r_{m}=p$.

Remark 2.2 (A special choice of the auxiliary weight functions $w_{0}, w_{m}$ ). If we choose

$$
w_{0}=W_{0} \quad \text { and } / \text { or } \quad w_{m}=W_{m}
$$

then the functions $B_{1}(x)$ and/or $B_{2}(x)$ become simpler in structure since (for $r_{0}<r$ and/or $r_{m}<p$ ) we have that

$$
\begin{aligned}
W_{0}^{-\frac{r_{0}}{r-r_{0}}}(t) w_{0}^{\frac{r}{r-r_{0}}}(t) & =W_{0}(t), \\
W_{m}^{-\frac{r_{m}}{p-r_{m}}}(t) w_{m}^{\frac{p}{p-r_{m}}}(t) & =W_{m}(t) .
\end{aligned}
$$

Consequently,

$$
\begin{aligned}
B_{1}(x)= & \left(\int_{x}^{b} t^{-(j+1) q} W(t)\left(\int_{0}^{t} W_{0}^{1-r_{0}^{\prime}}(s) d s\right)^{\frac{q}{r_{0}^{\prime}}} d t\right)^{\frac{r_{0}}{q}} \\
& \times\left(\int_{0}^{x} W_{0}(s) d s\right)^{\frac{r-r_{0}}{r}}, \\
B_{2}(x)= & \left(\int_{x}^{b} t^{(m-j-1) q} W(t)\left(\int_{0}^{t} W_{m}^{1-r_{m}^{\prime}}(s) d s\right)^{\frac{q}{r_{m}^{\prime}}} d t\right)^{\frac{r_{m}}{q}} \\
& \times\left(\int_{0}^{x} W_{m}(s) d s\right)^{\frac{p-r_{m}}{p}} .
\end{aligned}
$$

If we choose additionally $r_{0}=r$ and/or $r_{m}=p$, we have that

$$
\frac{W_{0}(t)}{w_{0}^{\tilde{p}}(t)} \equiv 1 \quad \text { and } / \text { or } \quad \frac{W_{m}(t)}{w_{m}^{\tilde{\tilde{r}}}(t)} \equiv 1
$$

and, e.g., for $r_{m}=p$ and $w_{m}=W_{m}$ the function $B_{2}(x)$ from (2.17) should be replaced by

$$
B_{2}^{*}(x)=\left(\int_{x}^{b} t^{(m-j-1) q} W(t)\left(\int_{0}^{t} W_{m}^{1-p^{\prime}}(s) d s\right)^{\frac{q}{p^{\prime}}} d t\right)^{\frac{p}{q}}
$$

with a similar change in (2.14).

Remark 2.3 (A different Hardy Inequality). If $p=q=r>1$ we can use the same basic idea as in (2.1)-(2.8) but substitute the Hardy Maximal Function 
inequality (cf. Hewitt and Stromberg [7]) for the Hardy inequality in (2.1)(2.8) which will allow us to substitute a general interval $I=[a, \infty),-\infty \leq a<$ $\infty$ for $[0, \infty)$. One form of the Maximal Function inequality is the following. Let $f$ be a nonnegative function in $L^{p}(I), 1<p<\infty$, and $J:=[x, c]$, $a \leq x \leq c<\infty$, a finite subinterval of $I$. For $x \in I$, define

$$
\mathfrak{M}[f](x):=\sup _{x \leq c<\infty} \frac{\int_{J} f(s) d s}{|J|} .
$$

Then

$$
\int_{I} \mathfrak{M}[f](x)^{p} d x \leq\left(\frac{p}{p-1}\right)^{p} \int_{I} f(x)^{p} d x .
$$

To implement the method using this inequality, we start by letting $J=J_{x} \equiv$ $[x, x+f(x)]$ in (2.1) where $f$ is a positive measurable function. Set $w_{0}=W_{0}^{\frac{1}{q}}$, $w_{m}=W_{m}^{\frac{1}{q}}$. Then Hölder's inequality gives as in (2.3) and (2.4)

$$
\begin{gathered}
\int_{J_{x}}|u(s)| d s \leq\left(\int_{J_{x}}|u(s)|^{r_{0}} W_{0}^{\frac{r_{0}}{q}}(s) d s\right)^{\frac{1}{r_{0}}}\left(\int_{J_{x}} W_{0}^{-\frac{r_{0}^{\prime}}{q}}(s) d s\right)^{\frac{1}{r_{0}^{\prime}}}, \\
\int_{J_{x}}\left|u^{(m)}(s) d s\right| \leq\left(\int_{J_{x}}\left|u^{(m)}(s)\right|^{r_{m}} W_{m}^{\frac{r_{m}}{q}}(s) d s\right)^{\frac{1}{r_{m}}}\left(\int_{J_{x}} W_{m}^{-\frac{r_{m}^{\prime}}{q}}(s) d s\right)^{\frac{1}{r_{m}^{\prime}}} .
\end{gathered}
$$

Now raise both sides of (2.1) to the $q$-th power and multiply by $W$. We get

$$
\begin{aligned}
W\left|u^{(j)}(x)\right|^{q} \leq & S_{1}(x)\left(f(x)^{-1} \int_{J_{x}}|u(s)|^{r_{0}} W_{0}^{\frac{r_{0}}{q}}(s) d s\right)^{\frac{q}{r_{0}}} \\
& +S_{2}(x)\left(C^{q} f(x)^{-1} \int_{J_{x}}\left|u^{(m)}(s)\right|^{r_{m}} W_{m}^{\frac{r_{m}}{q}}(s) d s\right)^{\frac{q}{r_{m}}}
\end{aligned}
$$

where

$$
\begin{aligned}
S_{1}(x) & =W(x) f(x)^{-(j+1) q+\frac{q}{r_{0}}}\left(\int_{J_{x}} W_{0}^{-\frac{r_{0}^{\prime}}{q}}(s) d s\right)^{\frac{q}{r_{0}^{\prime}}} \\
& =f(x)^{-j q} W(x)\left(f(x)^{-1} \int_{J_{x}} W_{0}^{-\frac{r_{0}^{\prime}}{q}}(s) d s\right)^{\frac{q}{r_{0}^{\prime}}}, \\
S_{2}(x) & =W(x) f(x)^{(m-j-1) q+\frac{q}{r_{m}}}\left(\int_{J_{x}} W_{m}^{-\frac{r_{m}^{\prime}}{q}}(s) d s\right)^{\frac{q}{r_{m}^{\prime}}} \\
& =W(x) f(x)^{(m-j) q}\left(f(x)^{-1} \int_{J_{x}} W_{m}^{-\frac{r_{m}^{\prime}}{q}}(s) d s\right)^{\frac{q}{r_{m}^{\prime}}} .
\end{aligned}
$$


Suppose now that $S_{1}(x), S_{2}(x)$ are bounded above on $I=[a, \infty)$ and assume that $u \in W^{m ; p, r}\left(I ; W_{0}, W_{m}\right)$. Next integrate both sides of $(2.22)$ over $I$. Since

$$
\begin{gathered}
\left(f(x)^{-1} \int_{J_{x}}|u(s)|^{r_{0}} W_{0}^{\frac{r_{0}}{q}}(s) d s\right) \\
\leq \sup _{a \leq c<b}\left((c-x)^{-1} \int_{x}^{c}|u(s)|^{r_{0}} W_{0}^{\frac{r_{0}}{q}}(s) d s\right) \\
\left(f(x)^{-1} \int_{J_{x}}\left|u^{(m)}(s)\right|^{r_{m}} W_{m}^{\frac{r_{m}}{q}}(s) d s\right) \\
\leq \sup _{a \leq c<b}\left((c-x)^{-1} \int_{x}^{c}\left|u^{(m)}(s)\right|^{r_{m}} W_{m}^{\frac{r_{m}}{q}}(s) d s\right),
\end{gathered}
$$

inequality (1.1) follows by a double application of the Hardy Maximal Inequality (with $p$ replaced by $\tilde{q} \equiv q / r_{0}$ or $\tilde{p} \equiv q / r_{m}$ ).

By this argument we have proven:

Theorem 2.2 Suppose $p=q=r$ then inequality (1.1) is true on an interval $I=[a, \infty)$ for all $u \in W^{m ; p, r}\left(I ; W_{0}, W_{m}\right)$ if the functions $S_{1}(x), S_{2}(x)$ are bounded on $I$.

Remark 2.4 (A change in the assumption (2.18)). The Hardy inequality (2.8) also holds for $1<\tilde{q}<\tilde{p}<\infty$, but in this case the necessary and sufficient condition (2.9) has to be replaced by

$$
A=\left(\int_{0}^{b}\left(\int_{t}^{b} H(s) d s\right)^{\frac{\tilde{\tilde{q}}}{\tilde{q}}}\left(\int_{0}^{t} K^{1-\tilde{p}^{\prime}}(s) d s\right)^{\frac{\tilde{\tau}}{\tilde{q}^{\prime}}} K^{1-\tilde{p}^{\prime}}(t) d t\right)^{\frac{1}{\tilde{r}}}<\infty .
$$

where

$$
\frac{1}{\tilde{r}}=\frac{1}{\tilde{q}}-\frac{1}{\tilde{p}}
$$

(see, e.g. [13, Theorem 1.15]).

Moreover we can suppose that $\tilde{q}$ satisfies

$$
0<\tilde{q}<\infty .
$$

If we choose $\tilde{p}, \tilde{q}$ according to (2.12), then from its definition the number 
$A$ has the form

$$
\begin{aligned}
& A_{1}=\left(\int_{0}^{b}\left(\int_{x}^{b} t^{-(j+1) q} W(t)\left(\int_{0}^{t} w_{0}^{1-r_{0}^{\prime}}(s) d s\right)^{\frac{q}{r_{0}^{\prime}}} d t\right)^{\frac{r}{r-q}}\right. \\
& \left.\times\left(\int_{0}^{x} W_{0}^{-\frac{r_{0}}{r-r_{0}}}(s) w_{0}^{\frac{r}{r-r_{0}}}(s) d s\right)^{\frac{r\left(q-r_{0}\right)}{r_{0}(r-q)}} W_{0}^{-\frac{r_{0}}{r-r_{0}}}(x) w_{0}^{\frac{r}{r-r_{0}}}(x) d x\right)^{\frac{r_{0}(r-q)}{r q}}
\end{aligned}
$$

since

$$
\tilde{r}=\frac{r_{0}(r-q)}{r q}, \quad \frac{\tilde{r}}{\tilde{q}}=\frac{r-q}{r}, \quad \frac{\tilde{r}}{\tilde{q}^{\prime}}=\frac{r\left(q-r_{0}\right)}{r_{0}(r-q) q} .
$$

In a similar way the choice of $\tilde{p}, \tilde{q}$ according to (2.15) leads to the expression

$$
\begin{aligned}
A_{2} & =\left(\int_{0}^{b}\left(\int_{x}^{b} t^{(m-j-1) q} W(t)\left(\int_{0}^{t} w_{m}^{1-r_{m}^{\prime}}(s) d s\right)^{\frac{q}{r_{m}^{\prime}}} d t\right)^{\frac{p}{p-q}}\right. \\
& \left.\times\left(\int_{0}^{x} W_{m}^{-\frac{r_{m}}{p-r_{m}}}(s) w_{0}^{\frac{p}{p-r_{m}}}(s) d s\right)^{\frac{p\left(q-r_{m}\right)}{r_{m}(p-q)}} W_{m}^{-\frac{r_{m}}{p-r_{m}}}(x) w_{m}^{\frac{p}{p-r_{0}}}(x) d x\right)^{\frac{r_{m}(p-q)}{p q}} .
\end{aligned}
$$

Using this version of Hardy's inequality we need not assume that $q \geq$ $\max \{p, r\}$ and can reformulate Theorem 2.1 as follows:

Theorem 2.3 The conclusion of Theorem 2.1 holds for $p, q, r$ in any of the following cases:

(i) The condition

$$
1<r \leq q<p
$$

holds and we suppose that the function $B_{1}(x)$ from (2.14) is bounded on $[0, b)$ and that $A_{2}<\infty$ where $A_{2}$ is given by the formula (2.24) and $r_{m}$ is chosen so that $r_{m} \neq q$.

(ii) The condition

$$
1<p \leq q<r
$$

holds and we suppose that the function $B_{2}(x)$ from (2.17) is bounded on $[0, b)$ and that $A_{1}<\infty$ where $A_{1}$ is given by formula (2.23) and $r_{0}$ is chosen so that $r_{0} \neq q$.

(iii) The condition

$$
0<q<\min \{p, r\}
$$

holds and we suppose that $A_{1}<\infty, A_{2}<\infty$ and that the auxiliary parameters are chosen so that $r_{0} \neq q, r_{m} \neq q$. 
(Notice that in case (iii) we can even allow $q$ to satisfy $0<q \leq 1$. Of course, in all cases, we assume that the conditions (2.19) are satisfied.)

Remark 2.5 (The case of an interval unbounded from below). Up to now we have assumed that the interval $[0, b)$ is bounded from below. Now let us consider an interval $(a, b)$ bounded from above; without loss of generality we can consider functions $u=u(t)$ defined on the interval $(a, 0],-\infty \leq a<0$. In this case we use (2.1) for the finite interval $I=[t, 0], a<t<0$, where now $|I|=|t|$, and start from the basic interpolation inequality

$$
\left|u^{(j)}(t)\right| \leq C|t|^{-(j+1)} \int_{t}^{0}|u(s)| d s+|t|^{(m-j-1)} \int_{t}^{0}\left|u^{(m)}(s)\right| d s .
$$

Proceeding analogously as in the case of the interval $[0, b)$, we finally obtain the following analogue of formula (2.5):

$$
\int_{a}^{0}\left|u^{(j)}(t)\right|^{q} W(t) d t \leq C_{1}\left(\mathcal{J}_{1}+\mathcal{J}_{2}\right)
$$

where now

$$
\begin{aligned}
\mathcal{J}_{1}=\int_{a}^{0}|t|^{-(j+1) q} W(t) & \left(\int_{t}^{0} w_{0}^{1-r_{0}^{\prime}}(s) d s\right)^{\frac{q}{r_{0}^{\prime}}} \\
& \times\left(\int_{t}^{0}|u(s)|^{r_{0}} w_{0}(s) d s\right)^{\frac{q}{r_{0}}} d t, \\
\mathcal{J}_{2}=\int_{a}^{0}|t|^{(m-j-1) q} W(t)\left(\int_{t}^{0} w_{m}^{1-r_{m}^{\prime}}(s) d s\right)^{\frac{q}{r_{m}^{\prime}}} & \times\left(\int_{t}^{0}\left|u^{(m)}(s)\right|^{r_{m}} w_{m}(s) d s\right)^{\frac{q}{r_{m}}} d t .
\end{aligned}
$$

In this case we have to use the "dual" form of Hardy's inequality (2.8), i.e.,

$$
\int_{a}^{0} H(t)\left(\int_{a}^{t} U(s) d s\right)^{\tilde{q}} d t \leq C_{0}\left(\int_{a}^{0} K(t) U^{\tilde{p}}(t) d t\right)^{\frac{\tilde{q}}{\tilde{p}}}
$$

which holds - provided $1<\tilde{p} \leq \tilde{q}<\infty$ - if and only if the function

$$
B(t)=\left(\int_{a}^{t} H(s) d s\right)^{\frac{1}{\tilde{q}}}\left(\int_{t}^{0} K^{1-\tilde{p}^{\prime}}(s) d s\right)^{\frac{1}{\tilde{p}^{\prime}}}
$$

is bounded on $(a, 0]$. 
Since all considerations are completely analogous to that of the case of the interval $[0, b]$, the formulation of the corresponding results is left to the reader. Roughly speaking, we have to replace in the formulas for $B_{1}(x), B_{2}(x), B_{1}^{*}(x)$, $B_{2}^{*}(x), A_{1}, A_{2}$, etc. the integral $\int_{t}^{b}$ by $\int_{a}^{t}$ and the integral $\int_{0}^{t}$ by $\int_{t}^{0}$.

Remark 2.6 If I in (1.1) is a compact interval, then (1.1) holds with weak conditions on the weights. If in (2.3) and (2.4) we take $w_{0}=W_{0}, w_{m}=W_{m}$, $r_{0}=r$, and $r_{m}=p$, then assuming $W_{0}^{1-r^{\prime}}, W_{m}^{1-p^{\prime}}$ are Lebesgue integrable on $I$ and substituting into (2.1) yields that

$$
\left|u^{(j)}(t)\right| \leq C\left\{\|u\|_{r, W_{0}}+\left\|u^{(m)}\right\|_{p, W_{m}}\right\}, \quad t \in I
$$

If also $W$ is Lebesgue integrable on I, then (1.1) follows from (2.28).

In Theorems 2.1 and 2.2 the inequality (1.1) is derived on either the interval $(0, b)$ or the interval $(a, 0)$. For integrals such as (2.14) to exist $W$ must be sufficiently small at 0 (see Example 2.1 below). We wish now to derive sufficient conditions for intervals of the form $(a, b), 0 \leq a<b \leq \infty$ where the weight functions do not have such constraints at the left endpoint. First we require a definition:

Definition 2.1 For $I=[a, b)$ we say that a is a regular point for (1.1) if for some $c<b, W, W_{0}^{1-r^{\prime}}$, and $W_{m}^{1-p^{\prime}}$ are Lebesgue integrable on $[a, c]$.

We next show how to prove a version of Theorem 2.1 where 0 is a regular point. Define for $c>0$ and $x \geq c$ :

$$
\begin{aligned}
B_{1, c}(x)= & \left(\int_{x}^{b} t^{-(j+1) q} W(t)\left(\int_{c}^{t} w_{0}^{1-r_{0}^{\prime}}(s) d s\right)^{\frac{q}{r_{0}^{\prime}}} d t\right)^{\frac{r_{0}}{q}} \\
& \times\left(\int_{c}^{x} W_{0}^{-\frac{r_{0}}{r-r_{0}}}(s) w_{0}^{\frac{r}{r-r_{0}}}(s) d s\right)^{\frac{r-r_{0}}{r}}
\end{aligned}
$$

and

$$
\begin{aligned}
B_{2, c}(x)= & \left(\int_{x}^{b} t^{(m-j-1) q} W(t)\left(\int_{c}^{t} w_{m}^{1-r_{m}^{\prime}}(s) d s\right)^{\frac{q}{r_{m}^{\prime}}} d t\right)^{\frac{r_{m}}{q}} \\
& \times\left(\int_{c}^{x} W_{m}^{-\frac{r_{m}}{p-r_{m}}}(s) w_{m}^{\frac{p}{p-r_{m}}}(s) d s\right)^{\frac{p-r_{m}}{p}}
\end{aligned}
$$

We define in a similar way $B_{1, c}^{*}(x), B_{2, c}^{*}, A_{1, c}$, and $A_{2, c}$. 
Theorem 2.4 Suppose $p, q, r, j, m, r_{0}$, and $r_{m}$ are as in Theorem 2.1 or Theorem 2.3 and let $W, W_{0}$, and $W_{m}$ be weight functions on $I=[0, b), 0<b \leq \infty$, which are regular at 0 and that for auxiliary weight functions $w_{0}, w_{m}$ on $[c, b)$ the functions $B_{1, c}(x)$ and $B_{2, c}(x), B_{1, c}^{*}(x)$ and $B_{2, c}^{*}$, and $A_{1, c}, A_{2, c}$ are bounded on $[c, b)$. Then the inequality (1.1) holds for every function $u \in$ $W^{m ; p, r}\left(I ; W_{0}, W_{m}\right)$ with a constant $C$ independent of $u$.

Proof. Define functions $\widetilde{w}_{0}, \widetilde{w}_{m}, \widetilde{W}$ on $[0, b)$ by

$$
\begin{aligned}
& \widetilde{w}_{0}(x)= \begin{cases}W_{0}^{\frac{r_{0} r^{\prime}}{r_{0}^{\prime}}}(x), & \text { on }[0, c] \\
w_{0}(x), & \text { on }[c, b),\end{cases} \\
& \widetilde{w}_{m}(x)= \begin{cases}W_{0}^{\frac{r_{m} p^{\prime}}{r_{m}^{\prime}}}(x), & \text { on }[0, c] \\
w_{m}(x), & \text { on }[c, b),\end{cases} \\
& \widetilde{W}(x)= \begin{cases}x^{(j+1) q}, & \text { on }[0, c] \\
W(x), & \text { on }[c, b) .\end{cases}
\end{aligned}
$$

Then a calculation gives that on $[0, c]$

$$
\begin{gathered}
\widetilde{w}_{0}^{1-r_{0}^{\prime}}=W_{0}^{-\frac{r_{0}}{r-r_{0}}} \widetilde{w}_{0}^{-\frac{r_{0}}{r-r_{0}}}=W_{0}^{1-r^{\prime}}, \\
\widetilde{w}_{m}^{1-r_{m}^{\prime}}=W_{m}^{-\frac{r_{m}}{p-r_{0}}} \widetilde{w}_{m}^{-\frac{r_{m}}{p-r_{m}}}=W_{m}^{1-p^{\prime}} .
\end{gathered}
$$

We consider explicitly only $B_{1, c}(x)$ and $B_{2, c}(x)$. The remaining cases are essentially the same. Let $\widetilde{B}_{1}(x)$ be as in $(2.14)$ where $W$ and $w_{0}$ are replaced by $\widetilde{W}$ and $\widetilde{w}_{0}$. Then from (2.31) and the definition of $\widetilde{W}$ a computation shows that the boundedness of $\widetilde{B}_{1}(x)$ on $[0, b)$ follows from that of $B_{1, c}(x)$ on $[c, b)$. Similarly let $\widetilde{B}_{2}(x)$ be as in $(2.17)$ where $W$ and $w_{m}$ are replaced by $\widetilde{W}$ and $\widetilde{w}_{m}$. As in the $B_{1}(x), \widetilde{B}_{1}(x)$ case, the boundedness of $\widetilde{B}_{2}(x)$ on $[0, b)$ follows. By Theorem 2.1, (1.1) holds on $[0, b)$ with $W$ replaced by $\widetilde{W}$. Thus

$$
\left(\int_{c}^{b} W(t)\left|u^{(j)}\right|^{q} d t\right)^{\frac{1}{q}} \leq\left\|u^{(j)}\right\|_{q, \widetilde{W}} \leq C\left(\|u\|_{r, W_{0}}+\left\|u^{(m)}\right\|_{p, W_{m}}\right)
$$

where the norms are on $[0, b)$. Since 0 is a regular endpoint, combining the last inequality with $(1.1)$ on $[0, c)$ yields $(1.1)$ on $[0, b)$.

We return now to the problem of proving (1.1) on an interval $I=[a, b)$, $a>0$. 
Theorem 2.5 Suppose that $p, q, r>1$ and the parameters $r_{0}, r_{m}$ satisfy (2.18) and (2.19) respectively. Let $m, j$ be integers $0 \leq j<m$ and let $W, W_{0}, W_{m}$, $w_{0}, w_{m}$ be weight functions on $I=[a, b), a>0$. Then the conclusions of Theorem 2.1 hold on $I$ in the following cases:

(i) $b=\infty, W, W_{0}, W_{m}, w_{0}, w_{m}$ are regular at $a$ and the functions

$$
\begin{aligned}
\widetilde{B}_{a, 1}(x) & =\left(\int_{x}^{b} t^{-(j+1) q} W(t+a)\left(\int_{a}^{t} w_{0}^{1-r_{0}^{\prime}}(s+a) d s\right)^{\frac{q}{r_{0}^{\prime}}} d t\right)^{\frac{r_{0}}{q}} \\
& \times\left(\int_{a}^{x} W_{0}^{-\frac{r_{0}}{r-r_{0}}}(s+a) w_{0}^{\frac{r}{r-r_{0}}}(s+a) d s\right)^{\frac{r-r_{0}}{r}}, \\
\widetilde{B}_{a, 2}(x) & =\left(\int_{x}^{b} t^{(m-j-1) q} W(t+a)\left(\int_{0}^{t} w_{m}^{1-r_{m}^{\prime}}(s+a) d s\right)^{\frac{q}{r_{m}^{\prime}}} d t\right)^{\frac{r_{m}}{q}} \\
& \times\left(\int_{a}^{x} W_{m}^{-\frac{r_{m}}{p-r_{0}}}(s+a) w_{m}^{\frac{p}{p-r_{m}}}(s+a) d s\right)^{\frac{p-r_{m}}{p}}
\end{aligned}
$$

are bounded on $I$.

(ii) $W, W_{0}, W_{m}$ are regular at $a$ and together with $w_{0}, w_{m}$ have extensions $\widehat{W}, \widehat{W}_{0}, \widehat{W}_{m}, \widehat{w}_{0}, \widehat{w}_{m}$ to $\hat{I}=[0, b)$ such that the functions $B_{1}(x)$ and $B_{2}(x)$ are bounded on $\hat{I}$ and

$$
\int_{I} W(t)(t-a)^{(m-1-j) q} d t<\infty .
$$

Proof. The proof of (i) is a direct application of Theorem 2.4. The weights $\widetilde{W}(t), \widetilde{W}_{0}(t), \widetilde{W}_{m}(t), \widetilde{w}_{0}(t), \widetilde{w}_{m}(t)$ defined by $W(t+a), W_{0}(t+a), W_{m}(t+a)$, etc. are regular at 0 . (2.32) and (2.33) correspond to (2.29) and (2.30). It follows that (1.1) holds with respect to $\widetilde{W}(t), \widetilde{W}_{0}(t), \widetilde{W}_{m}(t)$ on $[0, \infty)$. But the change of variable $t+a \rightarrow s$ shows that this inequality is equivalent to (1.1) with weights $W, W_{0}, W_{m}$ on $[a, \infty)$.

Next the assumptions of (ii) imply that (1.1) is valid for the extended weights on $[0, b)$. It follows that $(1.1)$ is true for functions in

$W_{L}^{m ; p, r}\left(I ; W_{0}, W_{m}\right)=\left\{u \in W^{m ; p, r}\left(I ; W_{0}, W_{m}\right): u^{(i)}(a+)=0, i=0, \ldots, m-1\right\}$

since functions in this class can be trivially extended to $[0, b)$ by defining them as 0 on $[0, a)$. Set

$$
G_{j}(u):=\left(\int_{I} W(t)\left|g_{u}^{(j)}(t)\right|^{q} d t\right)^{1 / q}<\infty
$$


where

$$
g_{u}(t):=\sum_{i=0}^{m-1} \frac{(t-a)^{i}}{i !} u^{(i)}(a+) .
$$

By $(2.34) G_{j}(u)$ is finite. Now let $u \in W^{m ; p, r}\left(I ; W_{0}, W_{m}\right)$. By the triangle inequality

$$
\left(\int_{I} W(t)\left|u^{(j)}(t)\right|^{q}\right)^{1 / q} d t \leq\left(\int_{I} W(t)\left|\left(u(t)-g_{u}(t)\right)^{(j)}\right|^{q} d t\right)^{1 / q}+G_{j}(u) .
$$

By definition $G_{j}(u)$ is a linear combination of the $u^{(i)}(a+), i=j, \ldots, m-1$. A Hölder inequality argument applied to the interpolation inequality (2.1) will give a family of inequalities each of the form

$$
\left|u^{(i)}(a+)\right| \leq K_{i}\left\{\left(\int_{a}^{c} W_{0}(t)|u(t)|^{r}\right)^{\frac{1}{r}} d t+\left(\int_{a}^{c} W_{m}(t)\left|u^{(m)}(t)\right|^{p}\right)^{\frac{1}{p}} d t\right\} .
$$

By addition and extension of the integration on the right hand side to $\hat{I}$, it follows that

$$
G_{j}(u) \leq K\left\{\left(\int_{I} W_{0}(t)|u(t)|^{r}\right)^{\frac{1}{r}} d t+\left(\int_{I} W_{m}(t)\left|u^{(m)}(t)\right|^{p}\right)^{\frac{1}{p}} d t\right\}
$$

Since $u-g_{u}(t) \in W_{L}^{m ; p, r}\left(I ; W_{0}, W_{m}\right)$ and (1.1) is true for these functions (1.1) follows at once for $u$ on $I$ by addition.

Remark 2.7 If we define " $\widetilde{B}_{a, 1}^{*}(x), \widetilde{B}_{a, 2}^{*}(x)$ " and " $\widetilde{A}_{a, 1}^{*}, \widetilde{A}_{a, 2}^{*}$ " as in (2.32) and (2.33), we can obtain versions of Theorem 2.5(i) for these cases. Also $B_{1}^{*}(x), B_{2}^{*}(x)$ or $A_{1}, A_{2}$ can be substituted for $B_{1}(x), B_{2}(x)$ in Theorem $2.5(i i)$. Hence in particular, we can prove Theorem 2.3 when $p, q, r$ satisfy (2.25), (2.26) or (2.27) on $I=[a, b)$ for $a>0$. Theorems 2.3 and 2.4 may also be easily modified to handle the case where $I$ is bounded above, i.e. $I=(a, b]$ where $-\infty \leq a<b<\infty$ and $b \neq 0$. The justifications of these various claims are left to the reader. However we will work out some of the consequences in the examples below.

Example 2.1 We take $b<\infty$ in Theorem 2.1 and set

$$
W(t)=t^{\alpha}, \quad W_{0}(t)=t^{\beta}, \quad W_{m}(t)=t^{\gamma},
$$

$\alpha, \beta, \gamma \in \mathbb{R}$. Suppose that (1.1) holds on $[0, b)$. We now derive a necessary condition. Let $u$ be a nontrivial $C^{\infty}$ function with compact support contained 
in $[0, b)$. We extend $u$ to $[0, \infty)$ by setting $u(x)=0$ on $(b, \infty)$. Then $(1.1)$ holds for $u$ and also for $u_{R}, R \geq 1$, defined by $u_{R}(t)=u(R t), 0<t \leq b$. (Note that the support of $u_{R}$ is contained in $[0, b)$.) Then $u_{R}^{(k)}(t)=R^{k} u^{(k)}(R t)$ and applying (1.1) to $u_{R}$ gives

$$
R^{j-\frac{\alpha+1}{q}}\left\|u^{(j)}\right\|_{q, t^{\alpha}} \leq C\left(R^{-\frac{\beta+1}{r}}\|u\|_{r, t^{\beta}}+R^{m-\frac{\gamma+1}{p}}\left\|u^{(m)}\right\|_{p, t^{\gamma}}\right),
$$

Since (2.36) holds for all $R \geq 1$ we obtain the necessary condition for (1.1) to hold that

$$
-j+\frac{\alpha+1}{q} \geq \min \left\{\frac{\beta+1}{r},-m+\frac{\gamma+1}{p}\right\} .
$$

If we had chosen $b=\infty$ with $R>0$, then we would have obtained the two necessary conditions

$$
\begin{aligned}
\min \left\{\frac{\beta+1}{r},-m+\frac{\gamma+1}{p}\right\} & \leq-j+\frac{\alpha+1}{q} \\
& \leq \max \left\{\frac{\beta+1}{r},-m+\frac{\gamma+1}{p}\right\} .
\end{aligned}
$$

Finally, if (1.1) is true on the interval $[a, \infty)$ as in Theorem 2.5(i) and $u$ is a function of compact support in $[a, \infty)$, we extend $u$ to $[0, \infty)$ by setting $u(x)=0$ on $[0, a)$. Then (1.1) holds for both $u$ and $u_{R}$ if $0<R \leq 1$ since the support of $u_{R}$ is contained in $[a, \infty)$. (2.36) then leads to the necessary condition

$$
-j+\frac{\alpha+1}{q} \leq \max \left\{\frac{\beta+1}{r},-m+\frac{\gamma+1}{p}\right\} .
$$

Next we check the sufficient conditions given by Theorem 2.1 with $b<\infty$. If we choose $r_{0}, r_{m}$ according to (2.19) and take

$$
w_{0}(t)=t^{r_{0}}, \quad w_{m}(t)=t^{r_{m}}
$$

then the functions $B_{1}(x)$ and $B_{2}(x)$ from $(2.14),(2.17)$ have the form

$$
\begin{aligned}
B_{1}(x)= & \left(\int_{x}^{b} t^{-(j+1) q+\alpha}\left(\int_{0}^{t} s^{\beta_{0}\left(1-r_{0}^{\prime}\right)} d s\right)^{\frac{q}{r_{0}^{\prime}}} d t\right)^{\frac{r_{0}}{q}} \\
& \times\left(\int_{0}^{x} s^{\frac{-\beta r_{0}+\beta_{0} r}{r-r_{0}}} d s\right)^{\frac{r-r_{0}}{r}},
\end{aligned}
$$




$$
\begin{aligned}
B_{2}(x)= & \left(\int_{x}^{b} t^{m-j-1) q+\alpha}\left(\int_{0}^{t} s^{\gamma_{0}\left(1-r_{m}^{\prime}\right)} d s\right)^{\frac{q}{r_{m}^{\prime}}} d t\right)^{\frac{r_{m}}{q}} \\
& \times\left(\int_{0}^{x} s^{\frac{-\gamma r_{m}+\gamma_{0} p}{p-r_{m}}} d s\right)^{\frac{r-r_{m}}{p}} .
\end{aligned}
$$

For $B_{1}(x)$ to be bounded on $[0, b)$ the following conditions are necessary:

(i) $\frac{-\beta r_{0}+\beta_{0} r}{r-r_{0}}+1>0 \Leftrightarrow \frac{\beta+1}{r}<\frac{\beta_{0}+1}{r_{0}}$,

(ii) $\beta_{0}\left(1-r_{0}^{\prime}\right)+1>0 \Leftrightarrow \frac{\beta_{0}+1}{r_{0}}<1$,

(iii) $\left[-(j+1) q+\alpha+1+\left(\beta_{0}\left(1-r_{0}^{\prime}\right)+1\right) \frac{q}{r_{0}^{\prime}}+1\right] \frac{r_{0}}{q}+\left(\frac{-\beta r_{0}+\beta_{0} r}{r-r_{0}}+1\right) \frac{r-r_{0}}{r} \geq$ $0 \Leftrightarrow-j+\frac{\alpha+1}{q} \geq \frac{\beta+1}{r}$.

Similarly $B_{2}(x)$ is bounded on $[0, b)$ if:

(i) $\frac{\gamma+1}{p}<\frac{\gamma_{0}+1}{r_{m}}$,

(ii) $\frac{\gamma_{0}+1}{r_{m}}<1$

(iii) $-j+\frac{\alpha+1}{q} \geq-m+\frac{\gamma+1}{p}$.

Thus Theorem 2.1 gives that (1.1) holds on $[0, b), b<\infty$, if

$$
\begin{gathered}
\frac{\beta+1}{r}<1, \quad \frac{\gamma+1}{p}<1, \\
-j+\frac{\alpha+1}{q} \geq \max \left\{\frac{\beta+1}{r},-m+\frac{\gamma+1}{p}\right\} .
\end{gathered}
$$

In a similar way, Theorem 2.1 gives that $(1.1)$ holds on $(0, \infty)$ if

$$
\begin{gathered}
\frac{\beta+1}{r}<1, \quad \frac{\gamma+1}{p}<1, \\
-j+\frac{\alpha+1}{q}=\frac{\beta+1}{r}=-m+\frac{\gamma+1}{p} .
\end{gathered}
$$


We can conclude therefore from (2.37) and (2.41) that if $\beta<r-1$ and $\gamma<p-1$ and

$$
\frac{\beta+1}{r}=-m+\frac{\gamma+1}{p},
$$

then

$$
-j+\frac{\alpha+1}{q} \geq \frac{\beta+1}{r}
$$

is a necessary and sufficient condition for inequality (1.1) with weights given by $(2.35)$ on $[0, b)$; likewise under these assumptions it follows from (2.38) that (2.42) is a necessary and sufficient condition for $(1.1)$ on $[0, \infty)$.

Example 2.2 Consider the above example on $I=[a, \infty)$ with $a>0$. The weights are regular at $a$. Further $\widetilde{W}(t), \widetilde{W}_{0}(t), \widetilde{W}_{m}(t)$ are regular at 0 . If we take $r_{0}=r, r_{m}=p, w_{0}=\widetilde{W}_{0}$, and $w_{m}=\widetilde{W}_{m}$. Using (2.21) and replacing $B_{1, a}, B_{2, a}$ by the upper bounds " $B_{1, a}^{*}, B_{2, a}^{*}$ " of $B_{1}^{*}(x), B_{2}^{*}(x)$ on $I$, we find that

$$
\begin{aligned}
& B_{1, a}^{*} \leq \sup _{x \in I}\left(\int_{x}^{\infty} t^{-(j+1) q}(t+a)^{\alpha}(t+a)^{\left(-\frac{\beta r^{\prime}}{r}+1\right) \frac{q}{r^{\prime}}}\right)^{\frac{r}{q}}, \\
& B_{2, a}^{*} \leq \sup _{x \in I}\left(\int_{x}^{\infty} t^{(m-j-1) q}(t+a)^{\alpha}(t+a)^{\left(-\frac{\gamma p^{\prime}}{p}+1\right) \frac{q}{p^{\prime}}}\right)^{\frac{p}{q}} .
\end{aligned}
$$

Since $t+a \sim t$ for large $t$, these are finite provided

$$
-j+\frac{\alpha+1}{q}<\min \left\{\frac{\beta+1}{r},-m+\frac{\gamma+1}{p}\right\} .
$$

Thus the inequality follows from (i) of Theorem 2.5 (cf. Remark 2.6). Here however we need not require that $\beta<r-1, \gamma<p-1$.

In (ii) of Theorem $2.5 \alpha, \beta, \gamma$ must satisfy (2.40). Additionally from (2.34) $G_{j}(u)<\infty$ if $-j+(\alpha+1) / q<-m+1$ which is satisfied since $(\gamma+1) / p<1$. Since the inequality on $[a, \infty)$ will continue to hold if $\alpha$ is diminished, the condition

$$
-j+\frac{\alpha+1}{q} \leq \min \left\{\frac{\beta+1}{r},-m+\frac{\gamma+1}{p}\right\}
$$

follows. So this part of Theorem 2.5 allows equality in $(2.45)$ if $(\gamma+1) / p<1$. Again from (2.39) the condition

$$
-j+\frac{\alpha+1}{q} \leq \frac{\beta+1}{r}
$$

is necessary and sufficient if (2.43) is true. 
Example 2.3 We take the same weights as in the above two examples on $I=[a, \infty)$ and $I=[o, \infty)$ with $q<\min \{p, r\}$. Let $w_{0}=W_{0}^{r_{0} / r}, w_{m}=W_{m}^{r_{m} / p}$, and $r_{0}=r_{m}$ be arbitrarily close to $q$. (Since we are going to end up with strict inequality constraints connecting $\alpha, \beta, \gamma, p, q, r$, this will mean that as a computational device, we can actually let $r_{0}, r_{m}$ take on the forbidden value $q$ without changing the final results.) We compute " $A_{1, a}$ " and " $A_{2, a}$ " in this way (cf. Remark 2.7), apply Theorem 2.5(i), and obtain finally that $\alpha, \beta, \gamma$ should satisfy (2.44). Since Theorem 2.3 does not apply to these weights on $[0, \infty)$ we cannot get cases of equality via Theorem 2.5(ii) as in the previous example.

Example 2.4 Theorems 2.1-2.3 will apply to the exponential weights $W(t)=$ $e^{\alpha t}, W_{0}(t)=e^{\beta t}, W_{m}(t)=e^{\gamma t}$ on $[0, \infty)$ if and only if both $\alpha<0$ and

$$
\frac{\alpha}{q}<\min \left\{\frac{\beta}{r}, \frac{\gamma}{p}\right\}
$$

However when $p=q=r, f(t)=1, W(t)=W_{0}(t)=W_{m}(t)=e^{\alpha t}$, simple calculations which we leave to the reader show that $S_{1}(x), S_{2}(x)$ are bounded on $[0, \infty)$ so that by Theorem $2.2(1.1)$ is valid in this case.

Remark 2.8 The results of the above examples are consistent with the conclusions reached for power-type or exponential-type weights in [4, Examples 1 and 2] where Besicovitch covering arguments were used to derive sum inequalities in $\mathbb{R}^{n}$. For $q \geq \max \{p, r\}$ our results are as a whole less general. When however $q<\min \{p, r\}$, Example 2.3 agrees precisely with [4, Example 1] but is more general since we can allow $0<q<1$.

\section{The Second Approach}

In this section we will deal only with the special case $j=0, m=1$ of (2.1). We will use the following interpolation inequality which holds for every function $u \in A C(J)$ with $J$ a compact interval in $\mathbb{R}$ :

$$
|u(t)| \leq\left(\int_{J} w_{0}(s) d s\right)^{-\frac{1}{r_{0}}}\left(\int_{J}|u(s)|^{r_{0}} w_{0}(s) d s\right)^{\frac{1}{r_{0}}}+\int_{J}\left|u^{\prime}(s)\right| d s
$$

where $t \in J$ is arbitrary, $w_{0}$ is a certain (auxiliary) weight function and $r_{0} \geq 1$ is an (auxiliary) parameter.

First we derive (3.1): For $u \in A C(J)$ let $c \in J$ be the point where $|u(s)|$ attains its minimum: $|u(c)|=\min _{s \in J}|u(s)|$. Then

$$
|u(c)|^{r_{0}} w_{0}(s) \leq|u(s)|^{r_{0}} w_{0}(s)
$$


for any $s \in J$. Integration with respect to $s$ over $J$ yields that

$$
|u(c)| \leq\left(\int_{J} w_{0}(s) d s\right)^{-\frac{1}{r_{0}}}\left(\int_{J}|u(s)|^{r_{0}} w_{0}(s) d s\right)^{\frac{1}{r_{0}}} .
$$

Since

$$
|u(t)|=\left|u(c)+\int_{c}^{t} u^{\prime}(s) d s\right| \leq|u(c)|+\int_{J}\left|u^{\prime}(s)\right| d s
$$

with $t \in J$ arbitrary, (3.1) follows at once because of (3.2).

Now we again consider functions $u=u(t) \in A C_{\text {loc }}(I)$ defined on an interval $I=[a, b)$ which is bounded from below, $-\infty<a<b \leq \infty$. (Note that in this approach we need not initially require that $a=0$.) Using (3.1) for the finite interval $[a, t] \subset I$, we obtain the inequality

$$
|u(t)| \leq\left(\int_{a}^{t} w_{0}(s) d s\right)^{-\frac{1}{r_{0}}}\left(\int_{a}^{t}|u(s)|^{r_{0}} w_{0}(s) d s\right)^{\frac{1}{r_{0}}}+\int_{a}^{t}\left|u^{\prime}(s)\right| d s .
$$

We then proceed as in Section 2: We choose an auxiliary parameter $r_{1}>1$ and an auxiliary weight function $w_{1}$ and estimate the second term on the right hand side of (3.3) by the Hölder inequality (2.4) (where we take $m=1$ ), take the $q$-th power of the resulting inequality, multiply both sides by $W(t)$ and integrate with respect to $t$ from $a$ to $b$. Finally, we obtain that

$$
\int_{a}^{b}|u(t)|^{q} W(t) d t \leq 2^{q-1}\left(\mathcal{J}_{1}+\mathcal{J}_{2}\right)
$$

where

$$
\begin{aligned}
& \mathcal{J}_{1}=\int_{a}^{b} W(t)\left(\int_{a}^{t} w_{0}(s) d s\right)^{-\frac{q}{r_{0}}}\left(\int_{a}^{t}|u(s)|^{r_{0}} w_{0}(s) d s\right)^{\frac{q}{r_{0}}} d t \\
& \mathcal{J}_{2}=\int_{a}^{b} W(t)\left(\int_{a}^{t} w_{1}^{1-r_{1}^{\prime}}(s) d s\right)^{-\frac{q}{r_{1}}}\left(\int_{a}^{t}\left|u^{\prime}(s)\right|^{r_{1}} w_{1}(s) d s\right)^{\frac{q}{r_{1}}} d t .
\end{aligned}
$$

Now we again use the Hardy inequality (2.8):

(a) The choice

$$
\begin{gathered}
\tilde{q}=\frac{q}{r_{0}}, \tilde{p}=\frac{r}{r_{0}}, \\
H(t)=W(t)\left(\int_{a}^{t} w_{0}(s) d s\right)^{-\frac{q}{r_{0}}}, \\
K(t)=W_{0}(t) w_{0}^{-\tilde{p}}(t), \\
U(t)=|u(t)|^{r_{0}} w_{0}(t),
\end{gathered}
$$


leads to the estimate

$$
\mathcal{J}_{1} \leq C_{0}\left(\int_{a}^{b}|u(t)|^{r} W_{0}(t) d t\right)^{\frac{q}{r}}
$$

which holds - provided $1<\tilde{p} \leq \tilde{q}<\infty$, i.e. $r \leq q-$ if and only if the function

$$
\begin{aligned}
B_{1}(x)= & \left(\int_{x}^{b} W(t)\left(\int_{a}^{t} w_{0}(s) d s\right)^{-\frac{q}{r_{0}}} d t\right)^{\frac{r_{0}}{q}} \\
& \times\left(\int_{a}^{x} W_{0}^{-\frac{r_{0}}{r-r_{0}}}(s) w_{0}^{\frac{r}{r-r_{0}}}(s) d s\right)^{\frac{r-r_{0}}{r}}
\end{aligned}
$$

is bounded on $I=[a, b)$.

(b) The choice

$$
\begin{aligned}
& \tilde{q}=\frac{q}{r_{1}}, \tilde{p}=\frac{p}{r_{1}} \\
& H(t)=W(t)\left(\int_{a}^{t} w_{1}^{1-r_{1}^{\prime}}(s) d s\right)^{\frac{q}{r_{1}^{\prime}}}, \\
& K(t)=W_{1}(t) w_{1}^{-\tilde{p}}(t), \\
& U(t)=\left|u^{\prime}(t)\right|^{r_{1}} w_{1}(t)
\end{aligned}
$$

leads to the estimate

$$
\mathcal{J}_{2} \leq C_{0}\left(\int_{a}^{b}\left|u^{\prime}(t)\right|^{p} W_{1}(t) d t\right)^{\frac{q}{p}}
$$

which holds - provided $1<\tilde{p} \leq \tilde{q}<\infty$, i.e. $p \leq q$ - if and only if the function

$$
\begin{aligned}
B_{2}(x)= & \left(\int_{x}^{b} W(t)\left(\int_{a}^{t} w_{1}^{1-r_{1}^{\prime}}(s) d s\right)^{\frac{q}{r_{1}^{\prime}}} d t\right)^{\frac{r_{1}}{q}} \\
& \times\left(\int_{a}^{x} W_{1}^{-\frac{r_{1}}{p-r_{1}}}(s) w_{1}^{\frac{p}{p-r_{1}}}(s) d s\right)^{\frac{p-r_{1}}{p}}
\end{aligned}
$$

is bounded on $I$.

The desired inequality (1.1) (for $j=0$ and $m=1$ ) now follows from (3.4), (3.5) and (3.7).

Summarizing we have the result: 
Theorem 3.1 Suppose that the numbers $p, q, r>1$ satisfy

$$
q \geq \max \{p, r\}
$$

Let $W_{0}, W_{1}$ and $W$ be weight functions on $I=[a, b),-\infty<a<b \leq \infty$. Suppose that for parameters $r_{0}, r_{1}$ with

$$
1 \leq r_{0}<r, \quad 1<r_{1}<p
$$

and for auxiliary weight functions $w_{0}, w_{1}$, the functions $B_{1}(x)$ and $B_{2}(x)$ from (3.6) and (3.8), respectively are bounded on $[a, b)$. Then the inequality

$$
\|u\|_{q, W} \leq C\left(\|u\|_{r, W_{0}}+\left\|u^{\prime}\right\|_{p, W_{1}}\right)
$$

holds for every function in $W^{1 ; p, r}\left(I ; W_{0}, W_{1}\right)$ with a constant $C$ independent of $u$.

Remark 3.1 In the same way as in Section 2 we can weaken the the conditions on the auxiliary parameters (allowing $r_{0}=r$ and/or $r_{1}=p$ ) and the auxiliary weights (choosing $w_{0}=W_{0}$ and/or $w_{1}=W_{1}$ ). We also can consider triples $p, q, r$ which do not satisfy (3.9) and derive a corresponding inequality also for the function $u$ defined on an interval bounded only from above. The results are analogous to those mentioned in Remarks 2.1 to 2.5 and in Theorem 2.3. The precise formulation is left to the reader.

Example 3.1 In the same way as in Example 2.1 we consider the interval $[0, \infty)$ and the weight functions $W(t)=t^{\alpha}, W_{0}(t)=t^{\beta}, W_{1}(t)=t^{\gamma}$. Choosing $w_{0}(t)=t^{\beta_{0}}, w_{0}(t)=t^{\gamma_{0}}$ as before, we obtain from (3.6) and (3.8) that

$$
\begin{aligned}
& B_{1}(x)=\left(\int_{x}^{\infty} t^{\alpha}\left(\int_{0}^{t} s^{\beta_{0}} d s\right)^{-\frac{q}{r_{0}}} d t\right)^{\frac{r_{0}}{q}}\left(\int_{0}^{x} s^{\frac{-\beta r_{0}+\beta_{0} r}{r-r_{0}}} d s\right)^{\frac{r-r_{0}}{r}}, \\
& B_{2}(x)=\left(\int_{x}^{\infty} t^{\alpha}\left(\int_{0}^{t} s^{\gamma_{0}\left(1-r_{1}^{\prime}\right)} d s\right)^{\frac{q}{r_{1}^{\prime}}} d t\right)^{\frac{r_{1}}{q}}\left(\int_{0}^{x} s^{\frac{-\gamma r_{1}+\gamma_{0} p}{p-r_{1}}} d s\right)^{\frac{p-r_{1}}{p}} .
\end{aligned}
$$

For $B_{1}(x)$ to be bounded on $[0, \infty)$ the following conditions are necessary:
(i) $\frac{-\beta r_{0}+\beta_{0} r}{r-r_{0}}+1>0$,
(ii) $\beta_{0}+1>0$,
(iii) $\left(\alpha-\left(\beta_{0}+1\right) \frac{q}{r_{0}}+1<0\right.$, 
(iv) $\left[\alpha+\left(\beta_{0}+1\right) \frac{q}{r_{0}}+1\right] \frac{r_{0}}{q}+\left(\frac{-\beta r_{0}+\beta_{0} r}{r-r_{0}}+1\right) \frac{r-r_{0}}{r}=0$,

i.e.,

$$
\frac{\beta_{0}+1}{r_{0}}>\frac{\beta+1}{r}, \quad \beta_{0}>-1, \quad \frac{\beta_{0}+1}{r_{0}}>\frac{\alpha+1}{q},
$$

and (from (iv))

$$
\frac{\alpha+1}{q}=\frac{\beta+1}{r} .
$$

The boundedness of $B_{2}(x)$ leads analogously to the conditions

$$
\frac{\gamma_{0}+1}{r_{1}}>\frac{\gamma+1}{p}, \quad \frac{\gamma_{0}+1}{r_{1}}<1, \quad \frac{\gamma_{0}+1}{r_{1}}>1+\frac{\alpha+1}{q}
$$

and

$$
\frac{\alpha+1}{q}=-1+\frac{\gamma+1}{r} .
$$

Thus this approach gives the same results as Example 2.1 (2.42) when $j=0$, $m=1$. In a similar fashion we can rederive the other examples of Section 2 . The details, however, will be left to the reader.

\section{Some Comments}

Remark 4.1 (Compactness of certain embeddings). Recall that the domain of our fundamental inequality (1.1)

$$
W^{m ; p, r}\left(I ; W_{m}, W_{0}\right) \equiv W^{m ; p, r}\left(W_{m}, W_{0}\right)
$$

consists of the set of all functions from $A C_{\text {loc }}^{m-1}[a, b)$ for which the norm

$$
\|u\|_{m ; p, r}=\left\|u^{(m)}\right\|_{p, W_{m}}+\|u\|_{r, W_{0}}
$$

is finite. This set is a weighted Sobolev space which is a Banach space if we assume additionally that

$$
W_{m}^{1-p^{\prime}} \in L_{\mathrm{loc}}^{1}[a, b), \quad W_{0}^{1-r^{\prime}} \in L_{\mathrm{loc}}^{1}[a, b)
$$

(see e.g., A. Kufner and B. Opic [8]).

Inequality (1.1) states that this weighted Sobolev space is continuously embedded into a weighted Lebesgue space:

$$
W^{m ; p, r}\left(W_{m}, W_{0}\right) \hookrightarrow L^{q}(W) .
$$


Under stronger assumptions on the weights and parameters of our problem we can show that the embedding (4.1) is compact.

For example, suppose that $q \geq \max \{p, r\}$ as in Theorem 2.1. Then (4.1) is compact if we additionally assume that

$$
B_{i}(x) \rightarrow 0 \text { for } x \rightarrow 0+\text { and for } x \rightarrow b-(i=1,2)
$$

where $B_{1}(x), B_{2}(x)$ are given by (2.14) and (2.17). Indeed, (4.2) guarantees that the embeddings realized through the Hardy inequality (2.8) are compact (see, e.g. [13, Theorem 7.3]). Let us note (cf. [13, Theorem 7.5]) that the embedding realized by the Hardy inequality (2.8) is automatically compact if $1<\tilde{q}<\tilde{p}<\infty$. It follows that the embedding (4.1) (whose continuity is guaranteed by Theorem 2.3(iii)) is compact without any additional conditions if $1<q<\min \{p, r\}$. Further if $q \geq \min \{p, r\}$ compactness results from the single condition (4.2) if $i=1$ and $1<r \leq q<p$ or if $i=2$ and $1<p \leq q<r$.

Example 4.1 In Example 2.2 the embedding is compact if the strict inequality conditions (2.44) hold. In this case a computation shows that $\widetilde{B}_{i}(x), i=1,2$ satisfy the conditions (4.2).

Remark 4.2 (A modification of the $j=0, m=1$ approach). The method described in Section 3 was based on the interpolation inequality (3.1) which was derived only in the special case $j=0, m=1$. Now we will introduce a modification of this inequality which will allow us to consider more general values of $j, m, 0 \leq j<m$. However this modification will force a restriction on the type of weight functions under consideration.

We will say that a weight function $w$ defined on an interval $[a, b)$ is regular if there exist constants $C>0$ and $\delta \in(0,1)$ such that for every subinterval $I \subset J \subset[a, b)$ with $|I| \geq \delta|J|$,

$$
\frac{\int_{J} w(t) d t}{\int_{I} w(t) d t} \leq C
$$

For a compact interval $I$ with length $|I|$ in the case $j=1, m=2$, and for a regular weight $w_{0}$, our basic interpolation inequality is:

$$
\left|u^{\prime}(t)\right| \leq C\left(\int_{I} w_{0}(s) d s\right)^{-1}|I|^{-1} \int_{I} w_{0}(s)|u(s)| d s+\int_{I}\left|u^{\prime \prime}(s)\right| d s
$$

where $t$ is arbitrary in $I$ and $C$ depends only on $w_{0}$. To derive (4.4) we consider an interval $I$ of length $L$ and denote by $I_{1}$ and $I_{3}$ the first and last third of $I$ 
respectively. Choosing $x_{1} \in I_{1}$ and $x_{3} \in I_{3}$ we have

$$
\frac{u\left(x_{3}\right)-u\left(x_{1}\right)}{x_{3}-x_{1}}=u^{\prime}(\xi)=u^{\prime}(t)-\int_{\xi}^{t} u^{\prime \prime}(s) d s
$$

for a certain $\xi \in\left[x_{1}, x_{3}\right]$ and an arbitrary $t \in I$. Since $\left|x_{3}-x_{1}\right| \geq \frac{L}{3}$ we have immediately from (4.5) that

$$
\left|u^{\prime}(t)\right| \leq \frac{3}{L}\left(\left|u\left(x_{3}\right)\right|+\left|u\left(x_{1}\right)\right|\right)+\int_{I}\left|u^{\prime \prime}(s)\right| d s .
$$

Multiplying this inequality by $w_{0}\left(x_{3}\right)$ and integration with respect to $x_{3}$ over $I_{3}$ yields that

$$
\left|u^{\prime}(t)\right| \leq \frac{3}{L}\left(\frac{\int_{I_{3}}|u(s)| w_{0}(s) d s}{\int_{I_{3}} w_{0}(s) d s}+\left|u\left(x_{1}\right)\right|\right)+\int_{I}\left|u^{\prime \prime}(s) d s\right|,
$$

Multiplying this inequality by $w_{0}\left(x_{1}\right)$ and integration with respect to $x_{1}$ over $I_{1}$ yields that

$$
\left|u^{\prime}(t)\right| \leq \frac{3}{L}\left(\frac{\int_{I_{3}}|u(s)| w_{0}(s) d s}{\int_{I_{3}} w_{0}(s) d s}+\frac{\int_{I_{1}}|u(s)| w_{0}(s) d s}{\int_{I_{1}} w_{0}(s) d s}\right)+\int_{I}\left|u^{\prime \prime}(s)\right| d s,
$$

and thus

$$
\left|u^{\prime}(t)\right| \leq \frac{3}{L}\left(\int_{I} w_{0}\right)^{-1}\left(\int_{I}|u| w_{0}\right)\left(\frac{\int_{I} w_{0}}{\int_{I_{3}} w_{0}}+\frac{\int_{I} w_{0}}{\int_{I_{1}} w_{0}}\right)+\int_{I}\left|u^{\prime \prime}\right| .
$$

Using the regularity condition (4.3) with e.g., $\delta=1 / 3$, (4.4) follows immediately.

If we now use (4.4) for $I=[a, t]$ for functions $u=u(x)$ defined on $[a, b)$ with $a<b \leq \infty$ or for $I=[t, b)$ for functions defined on $(a, b],-\infty \leq a<b<\infty$ we can proceed just as in Sections 2 and 3 to derive additional sufficient conditions for inequality (1.1).

\section{References}

[1] R. C. Brown and D. B. Hinton, Sufficient conditions for weighted inequalities of sum form, J. Math. Anal. Appl., 112 (1985), 563-578.

[2] R. C. Brown and D. B. Hinton, Sufficient conditions for weighted Gabushin inequalities, Časopis Pěst. Mat., 111 (1986), 113-121. 
[3] R. C. Brown and D. B. Hinton, Necessary and sufficient conditions for one variable weighted interpolation inequalities, Proc. London Math. Soc., 35(2) (1987), 439-453.

[4] R. C. Brown and D. B. Hinton, Weighted interpolation inequalities of sum and product form in $R^{n}$, J. London Math Soc., 56(3) (1988), 261-280.

[5] R. C. Brown and D. B. Hinton, Weighted interpolation inequalities and embeddings in $R^{n}$, Canad. J. Math., 47 (1990), 959-980.

[6] B. Curgus and T. Read, Discretness of the spectrum of second-order differential operators and associated embedding theorems, (to appear).

[7] E. Hewitt and K. Stromberg, Real and Abstract Analysis, Springer-Verlag, Berlin-Heidelberg-New York, 1969.

[8] A. Kufner and B. Opic, How to define reasonably weighted Sobolev spaces, Comment. Math. Univ. Carolin., 25 (1984), 537-554.

[9] P. I. Lizorkin and M. O. Otelbaev, Imbedding and compactness theorems for Sobolev type spaces with weights I, Mat. Sb. (N. S.), 108(150), 358-377, (in Russian).

[10] P. I. Lizorkin and M. O. Otelbaev, Imbedding and compactness theorems for Sobolev type spaces with weights II, Mat. Sb. (N. S.), 112(154), no. 1, (1980), 56-85, (in Russian).

[11] V. G. Maz'ja, Sobolev Spaces, Springer-Verlag, Berlin-Heidelberg-New York, 1985.

[12] R. Oinnarov, On weighted norm inequalities with three weights, J. London Math. Soc., (2) 48 (1993), 103-116.

[13] B. Opic and A. Kufner, Hardy-type Inequalities, Longman Scientific and Technical, Harlow, Essex, UK, 1990. 